\title{
Evidence for a Switching Mechanism in the Invasion of Erythrocytes by Plasmodium falciparum
}

Stephen A. Dolan, Louis H. Miller, and Thomas E. Wellems

Laboratory of Parasitic Diseases, National Institutes of Health, Bethesda, Maryland 20892

\begin{abstract}
The human malaria parasite Plasmodium falciparum demonstrates variability in its dependence upon erythrocyte sialic acid residues for invasion. Some lines of $\boldsymbol{P}$. falciparum invade neuraminidase-treated or glycophorin-deficient red blood cells poorly, or not at all, while other lines invade such cells at substantial rates. To explore the molecular basis of non-sialic acid dependent invasion, we selected parasite lines from a clone (Dd2) that initially exhibited low invasion of neuraminidasetreated erythrocytes. After maintaining Dd2 for several cycles in neuraminidase-treated erythrocytes, parasite lines were recovered that invaded both untreated and neuraminidasetreated erythrocytes at equivalently high rates (Dd2/NM). The change in phenotype was maintained after removal of selection pressure. Four subclones of Dd2 were isolated and each readily converted from sialic acid dependence to non-sialic acid dependence during continuous propagation in neuraminidase-treated erythrocytes. The neuraminidase-selected lines and the Dd2 clone demonstrated identical restriction fragment length polymorphism markers indicating that the Dd2 clone was not contaminated during the selection process. Parasite proteins that bound to neuraminidase-treated and untreated erythrocytes were indistinguishable among the parent Dd2 clone and the neuraminidase-selected lines. The ability of the Dd2 parasite to change its invasion requirements for erythrocyte sialic acid suggests a switch mechanism permitting invasion by alternative pathways. (J. Clin. Invest. 1990. 86:618624.) Key words: malaria • erythrocytes $\bullet$ sialic acid
\end{abstract}

\section{Introduction}

Recently several studies have focused on erythrocyte sialic acid and its role in invasion of erythrocytes by the human malaria parasite Plasmodium falciparum (1-7). Some studies have shown that different lines of $P$. falciparum vary in their dependence on erythrocyte sialic acid for effective invasion of red blood cells (8-10). Cleavage of sialic acid from erythrocytes with neuraminidase treatment greatly reduces erythrocyte invasion by some parasite lines, but produces only modest reduction by others $(1,2,6,8-10)$. Erythrocytes genetically deficient in glycophorin molecules, which contain the majority of erythrocyte sialic acid (11), have confirmed this diversity in the invasion characteristics of $P$. falciparum $(1,5,9,12)$.

Address correspondence to Dr. Stephen A. Dolan, Laboratory of Parasitic Diseases, National Institutes of Health, Building 4, Room 126, 9000 Rockville Pike, Bethesda, MD 20892.

Received for publication 22 January 1990 and in revised form 21 March 1990.

The Journal of Clinical Investigation, Inc.

Volume 86, August 1990, 618-624
Specific proteins synthesized by the parasite have been found to bind to neuraminidase-sensitive residues on the red cell surface $(13,14)$. The binding of these proteins has been correlated with the ability of the sialic acid-dependent parasite to invade susceptible erythrocytes. Parasite or erythrocyte molecules involved in non-sialic acid dependent pathways have not been identified. A trypsin-sensitive erythrocyte molecule, distinct from glycophorin $\mathrm{A}$, has been implicated as being involved in invasion by some parasites (9). To this end, we have begun to explore the effect of selection pressure on parasite invasion in vitro.

In this report we show that $P$. falciparum clones can be grouped into two categories according to their ability to invade sialic acid-deficient erythrocytes. Type I parasites invade and grow in neuraminidase-treated erythrocytes, although at reduced rates as compared with untreated red cells. Type II parasites can be subdivided into two categories, II-A and II-B. With continued cultivation in neuraminidase-treated erythrocytes, type II-A parasites disappear from culture, demonstrating their complete dependence on sialic acid for erythrocyte invasion. Type II-B parasites eventually produce a population that is able to effectively invade neuraminidase-treated erythrocytes; this subpopulation emerges during continuous propagation in neuraminidase-treated erythrocytes. The type II-B phenotypic change is reproducible in the parent clone and in multiple subclones of the parent. Furthermore, the change persists in the absence of selection pressure, suggesting that a switch occurs in the expression of a gene or group of genes of type II-B parasites.

\section{Methods}

P. falciparum clones. P. falciparum clones Dd2 (15), HB3 (16), 3D7 (17), 7 G8 (18), Camp/A1 $(8,9)$, and FCR-3/A2 (19) were cultivated in vitro according to the methods of Trager and Jensen (20). Subclones of Dd2 were obtained by limiting dilution (21). To avoid variability in erythrocytes from different individuals, $\mathrm{O}^{+}$erythrocytes from a single individual (S.A.D.) were used in all cultures and experiments. Cultures were maintained at 5\% hematocrit in RPMI 1640 (Gibco Laboratories, Grand Island, NY) to which $24 \mathrm{mM}$ Hepes, $360 \mu \mathrm{M}$ hypoxanthine, 24 $\mathrm{mM} \mathrm{NaHCO}, 10 \mu \mathrm{g} / \mathrm{ml}$ of gentamicin and $10 \%$ heat-inactivated, pooled, human $\mathrm{A}^{+}$serum were added. Incubation was at $37^{\circ} \mathrm{C}$ under $3 \% \mathrm{O}_{2} / 6 \% \mathrm{CO}_{2} / 91 \% \mathrm{~N}_{2}$.

Neuraminidase treatment of erythrocytes. Erythrocytes were collected by venipuncture into citrate-phosphate-dextrose solution, pelleted from the plasma, washed in 5 vol of RPMI 1640, centrifuged, and resuspended to $50 \%$ hematocrit in either complete medium or RPMI 1640 at $4^{\circ} \mathrm{C}$. Neuraminidase treatment of erythrocytes was performed as described $(8,9)$. Neuraminidase (from Vibrio cholera; Gibco Laboratories) was added directly to the washed erythrocytes at $10-50 \mathrm{U} / \mathrm{ml}$. Erythrocytes were incubated rocking at $37^{\circ} \mathrm{C}$ for $60-90 \mathrm{~min}$, pelleted, washed in $10 \mathrm{vol}$ of RPMI 1640 , resuspended at $50 \%$ hematocrit with either complete medium or RPMI 1640 , and stored at $4^{\circ} \mathrm{C}$.

Invasion assays. Parasite cultures were synchronized with 5\% sorbitol (22) and expanded to 3-5\% late trophozoite forms in $25-50-\mathrm{ml}$ 
cultures. The erythrocytes were pelleted, resuspended to $50 \%$ hematocrit in complete medium, and layered over either a $65 \%$ Percoll/complete medium cushion or a Percoll/sorbitol gradient $(23,24)$. Late stage forms were recovered (50-90\% infected red blood cells), washed with RPMI 1640, counted with a hemocytometer, and resuspended in complete medium at $1-5 \times 10^{7}$ parasites $/ \mathrm{ml}$. Invasion assays were performed in 96-well, flat-bottomed microtiter plates (Costar, Cambridge, MA). $100 \mu \mathrm{l}$ of the parasite suspension was inoculated into 100 $\mu l$ of target erythrocytes at $2-5 \times 10^{8}$ erythrocytes $/ \mathrm{ml}$ in complete medium. A 1:10-1:20 ratio of parasites/target erythrocytes was used. Assays were incubated at $37^{\circ} \mathrm{C}$ for $18-24 \mathrm{~h}$. The number of ring forms per 1,000 erythrocytes was estimated from blood films. Individual assays were performed in duplicate and the invasion rates were taken as the average result. Invasion rates for neuraminidase-treated erythrocytes were recorded as percent of control erythrocyte invasion rates.

Growth assays. Parasite cultures were grown to $\sim 5 \%$ asynchronous parasitemia in normal erythrocytes (5-ml volume). From this culture an inoculum was delivered to fresh 5-ml cultures containing either untreated or neuraminidase-treated erythrocytes (initial parasitemia $0.1-0.5 \%$ ). Parasitemia was estimated daily from the number of parasitized erythrocytes per 1,000 erythrocytes.

Erythrocyte binding assays. 25-50-ml cultures were synchronized with sorbitol and allowed to reach 3-5\% late trophozoite forms. After enrichment on a Percoll gradient, the trophozoites were returned to methionine-cysteine-deficient complete medium (Select-Amine Kit; Gibco Laboratories) at 1-3 $\times 10^{8}$ parasites $/ \mathrm{ml}$ and labeled with $100-200 \mu \mathrm{Ci} / \mathrm{ml}$ of radiolabeled methionine and cysteine (Trans ${ }^{35} \mathrm{~S}$ Label; ICN Radiochemicals, Irvine, CA). After incubation for 12-20 h the culture was centrifuged $\left(12,000 \mathrm{~g}\right.$ for $10 \mathrm{~min}$ at $\left.4^{\circ} \mathrm{C}\right)$. The supernatant was frozen at $-70^{\circ} \mathrm{C}$. The pellet, containing schizonts, free merozoites, and cellular debris, was resuspended in $3 \mathrm{ml}$ of RPMI 1640 in a $15-\mathrm{ml}$ Corex centrifuge tube and sonicated on a wet ice bath. Three cycles of sonication ( $30 \mathrm{~s}$ on/30 $\mathrm{s}$ off) were completed at an output control setting of 100 (sonic disruptor; Tekmar Co., Cincinnati, $\mathrm{OH}$ ). The sonicate was centrifuged at $12,000 \mathrm{~g}$ for $20 \mathrm{~min}$ at $4^{\circ} \mathrm{C}$. The supernatant was frozen at $-70^{\circ} \mathrm{C}$ until use. $500 \mu \mathrm{l}$ of supernatant and $250 \mu \mathrm{l}$ of sonicate were mixed with $100 \mu \mathrm{l}$ of packed erythrocytes. Erythrocyte binding assays were performed as described by Haynes et al. except protease inhibitors were not included (25).

Southern blot analyses. DNA was prepared from saponin-lysed, parasitized erythrocytes (26) and separated over cesium chloride gradients according to standard procedures (27). Restriction endonuclease digests were performed with enzymes from Bethesda Research Laboratories (Gaithersburg, MD) and New England Biolabs (Beverly, MA) according to manufacturers' recommendations. Restriction fragments were fractionated in agarose gels and transferred to nylon membranes for hybridization (28). The pC4.H32 probe, obtained from a size-fractionated Hinf I $P$. falciparum library (29), was used to "fingerprint" repetitive elements in the genome.

\section{Results}

Variation among clones in sialic acid requirements. To assess sialic acid requirements for invasion, six $P$. falciparum clones were evaluated for their ability to invade neuraminidasetreated erythrocytes (Fig. 1). These invasion assays revealed that parasites could be classified into two groups according to their dependence on sialic acid residues. Type I parasites (HB3, 3D7, and 7G8) invaded neuraminidase-treated erythrocytes at $30-60 \%$ of the rate in control erythrocytes. Type II parasites (FCR-3/A2, Camp/A1, and Dd2) invaded neuraminidase-treated erythrocytes at low rates compared with control (5-15\%). Low residual invasion rates of Dd2, FCR-3/A2, and Camp/A1 into neuraminidase-treated erythrocytes may, in part, be accounted for by the presence of untreated erythrocytes that were carried with the parasite inoculum after enrich-

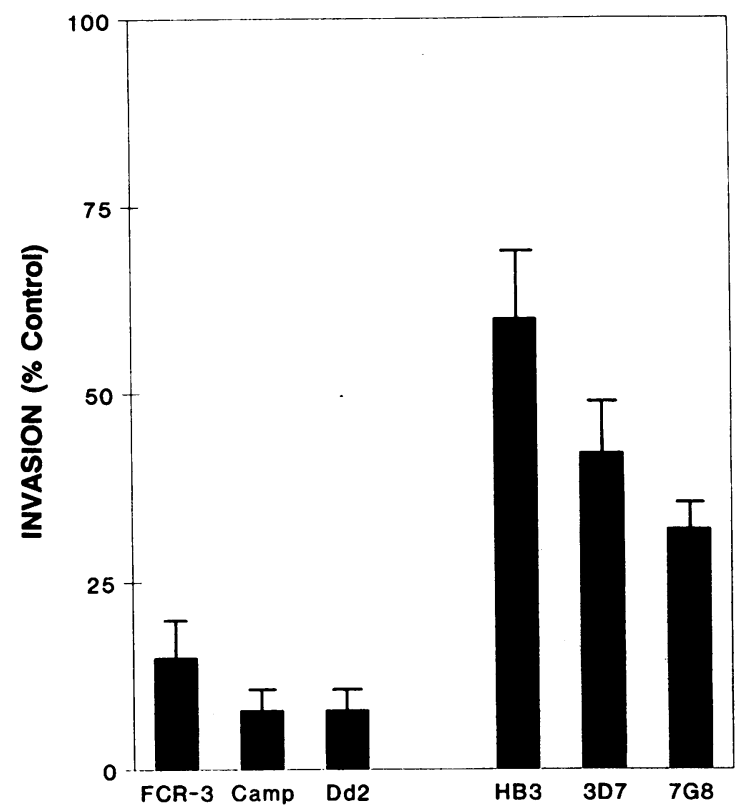

Figure 1. Invasion of neuraminidase-treated erythrocytes by type I and type II $P$. falciparum parasites. Results are an average of three separate invasion assays and are presented as the percentage of invasion into neuraminidase-treated erythrocytes relative to invasion of untreated erythrocytes. In all experiments the invasion rate of untreated erythrocytes was $>3.5 \%$. Error bars indicate SEM.

ment. Preabsorption of the sera used in the culture medium with neuraminidase-treated erythrocytes did not affect results of the invasion assays with Dd2 and HB3. The decreased invasion, therefore, was not related to antibody binding of neuraminidase-treated erythrocytes.

To study the growth characteristics of type I and type II parasites, the six parasite clones were each placed into parallel cultures with untreated and neuraminidase-treated erythrocytes and propagated for several days (Fig. 2). Type I parasites (HB3, 3D7, and 7G8) grew in neuraminidase-treated erythrocytes at approximately one-half to one-third of the rate in control cells. In contrast, type II parasites subdivided based on their growth in neuraminidase-treated erythrocytes. Type II-A parasites (FCR-3/A2 and Camp/A1) exhibited complete dependence on erythrocyte sialic acid residues. When attempts were made to propagate type II-A parasites in neuraminidasetreated red blood cells, the parasites were lost from culture and could not be recovered despite maintenance of the culture for 4 wk. However, clone Dd2 (type II-B) exhibited novel behavior when propagated in neuraminidase-treated erythrocytes. After the inoculation of Dd2 into neuraminidase-treated erythrocytes, parasites were no longer detectable by thin-film blood films after two to three growth cycles. However, after 10-12 d in continuous culture, a population of parasites emerged that was capable of invasion and growth in neuraminidase-treated erythrocytes (Dd2/NM1). The experiment was repeated and a second line of parasites capable of invasion and growth in neuraminidase-treated red cells was again obtained (Dd2/NM2).

Invasion rates of Dd2/NM1 and Dd2/NM2 into neuraminidase-treated erythrocytes were nearly equivalent to the rates of invasion into untreated erythrocytes (Fig. $3 \mathrm{~A}$ ). Fur- 

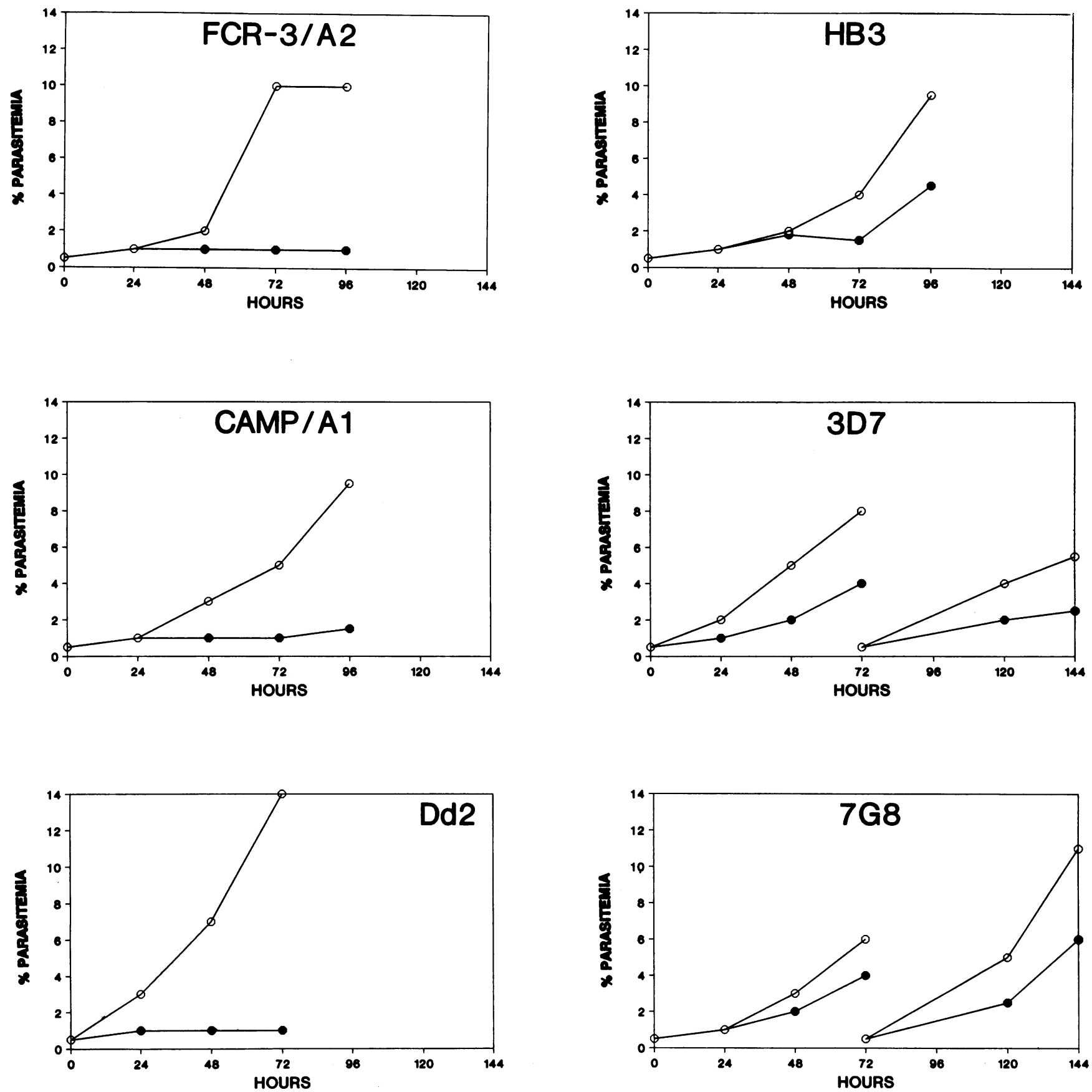

Figure 2. Propagation of $P$. falciparum clones in untreated and neuraminidase-treated erythrocytes. Clones 3D7 and 7G8 were diluted to $0.5 \%$ parasitemia at $72 \mathrm{~h}$ and cultivated for $144 \mathrm{~h}$. ๑, Neuraminidase-treated erythrocytes; $\circ$, untreated erythrocytes.

thermore, in both untreated and neuraminidase-treated erythrocytes, Dd2/NM1 was able to grow at rates comparable to those of Dd2 in untreated erythrocytes (Fig. 3 B). Such behavior by Dd2/NM1 and Dd2/NM2 contrasts with the lower rates observed for type I clones in neuraminidase-treated erythrocytes (Fig. 1).

The Dd2/NM1 and Dd2/NM2 phenotypes were found to be stable in culture with untreated normal erythrocytes. After their selection in neuraminidase-treated erythrocytes, both lines were propagated in untreated erythrocytes for at least 2 wk (eight cycles) and then reexamined for invasion into neur- aminidase-treated erythrocytes. Despite removal of selection pressure, the Dd2/NM1 and Dd2/NM2 lines continued to invade neuraminidase-treated erythrocytes and untreated erythrocytes at similar rates.

To confirm that the Dd2/NM1 and Dd2/NM2 lines had been selected from a clonal population, Dd 2 was subcloned by limiting dilution. Four subclones were examined and each of these subclones (Dd2/R7, Dd2/R8, Dd2/R9, and Dd2/R14) exhibited the same phenotypic switch as the $\operatorname{Dd} 2$ clone. Growth assays revealed an initial inability of the inocula to expand in neuraminidase-treated erythrocytes; however, 


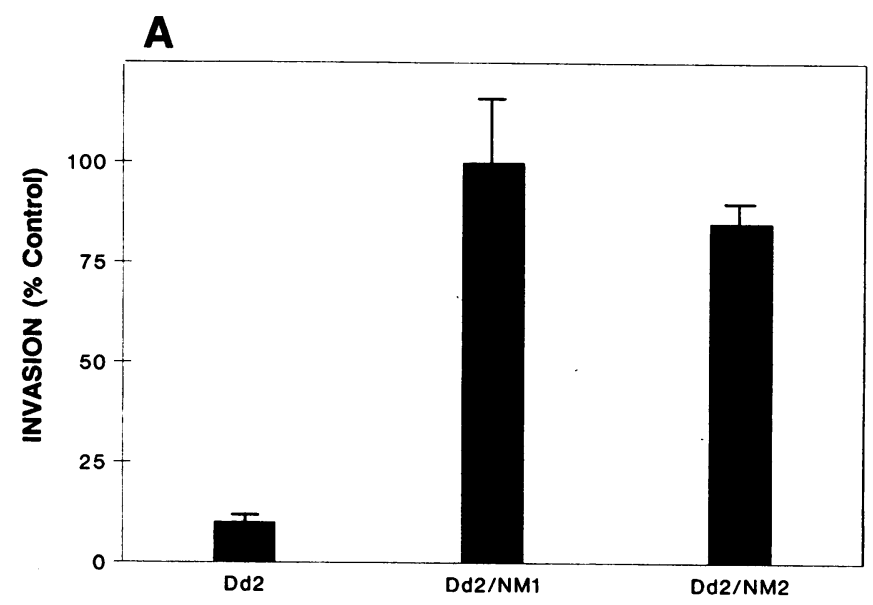

B

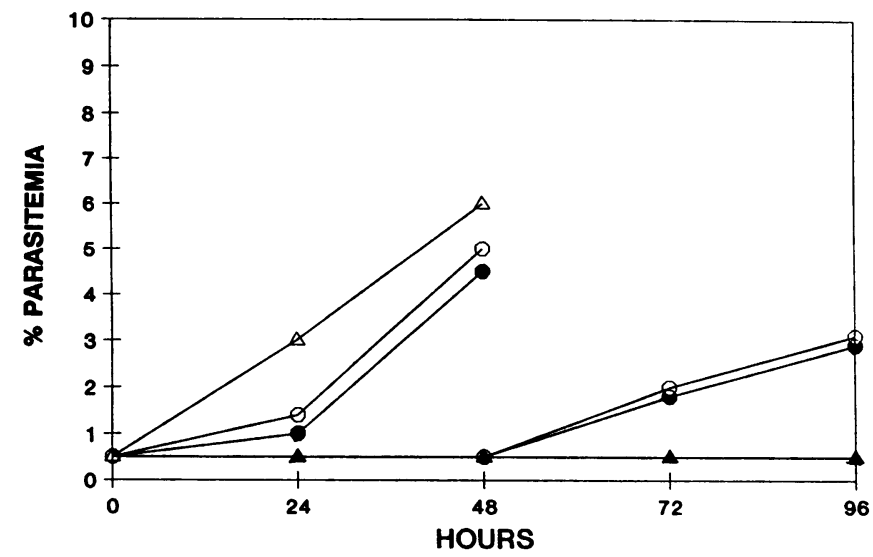

Figure 3. Invasion and growth of the Dd2/NM1 and Dd2/NM2 lines. $A$, Invasion rates of $\mathrm{Dd} 2, \mathrm{Dd} 2 / \mathrm{NM} 1$, and $\mathrm{Dd} 2 / \mathrm{NM} 2$ into neuraminidase-treated and untreated erythrocytes. The results are an average of three separate invasion assays for Dd2 and Dd2/NM1 and two separate invasion assays for Dd2/NM2 presented as the percentage of invasion into neuraminidase-treated erythrocytes relative to invasion into untreated erythrocytes. In all experiments the invasion rate of untreated erythrocytes was $>3.5 \%$. Error bars indicate SEM. $B$, Growth of Dd2 and Dd2/NM1 in neuraminidase-treated and untreated erythrocytes. Dd2/NM1 parasites were diluted 1:10 at $48 \mathrm{~h}$. Propogation of Dd2 in untreated cells was discontinued at $48 \mathrm{~h}$. Triangles, Dd2; octagons, Dd2/NM1; open symbols, untreated erythrocytes; solid symbols, neuraminidase-treated erythrocytes.

maintenance in culture for 10-12 d produced lines from each subclone capable of growth in neuraminidase-treated erythrocytes (Dd2/NM7, Dd2/NM8, Dd2/NM9, and Dd2/NM14). Therefore, each subclone from $\mathrm{Dd} 2$ had the intrinsic ability to convert from sialic acid dependence to sialic acid independence.

To confirm the clonal integrity of Dd2 and the Dd2/NM lines, the genomic structure of the parasites was examined by probing restriction digests with single-copy and repetitive sequences from the genome. Fig. 4 shows hybridization patterns obtained with pC4.H32, a recombinant clone that detects interspersed repetitive elements on chromosomes of the parasite. Clones HB3, 3D7, 7G8, FCR-3/A2, Camp/A1, and Dd2 each exhibited unique patterns of hybridization ("fingerprints"). By comparison, the subclones of Dd2 and the NM lines all produced patterns identical to that of the parent Dd2 clone. Restriction fragment length polymorphism analysis likewise revealed no detectable differences in the $\mathrm{Dd} 2$ family. 30 separate plasmid and oligonucleotide probes hybridized against $18 \mathrm{sep}$ arate restriction endonuclease digests revealed no polymorphisms among Dd2, Dd2/NM1, and Dd2/NM2 (data not shown).

Erythrocyte binding assays. Erythrocyte binding assays with soluble proteins from culture supernatants and parasiteinfected cellular sonicates of Dd2, Dd2/NM1, and Dd2/NM2 parasites were performed. Comparison of protein binding to untreated and neuraminidase-treated erythrocytes was made in an attempt to explain the phenotypic differences among the parasites. Culture supernatant mixed with normal erythrocytes and neuraminidase-treated erythrocytes revealed similar patterns of bound radiolabeled proteins among the three lines (Fig. $5 \mathrm{~A}$, lanes $2,5,8$ ). Some radiolabeled proteins, such as a 175-, a 165-, and a 125-kD bound preferentially to untreated erythrocytes. With neuraminidase-treated erythrocytes, however, a $140-\mathrm{kD}$ protein produced the most prominent eluted band and bound more strongly to neuraminidase-treated erythrocytes than untreated erythrocytes. Neither Dd2/NM1 nor Dd2/NM2 demonstrated alterations in protein-binding profiles that could account for their acquired ability to invade neuraminidase-treated erythrocytes.

Sonicates of parasite-infected cells demonstrated patterns of bound radiolabeled proteins to either untreated or neuraminidase-treated erythrocytes that were also similar among the three lines (Fig. $5 \mathrm{~B}$ ). The most prominent protein from the cellular sonicate bound to untreated erythrocytes was at 165 $\mathrm{kD}$; less intense bands were seen at 175,140 , and $125 \mathrm{kD}$. As with the culture supernatants, a $140-\mathrm{kD}$ radiolabeled protein was the most prominent protein eluted from neuraminidasetreated erythrocytes. The sonicates did not show any differences in the patterns of radiolabeled protein bound by $\mathrm{Dd} 2$ / NM1, Dd2/NM2, and Dd2.

\section{Discussion}

In this work $P$. falciparum parasites have been classified into two categories based on their requirements for erythrocyte sialic acid residues for invasion. Type I parasites, represented by clones HB3, 3D7, and 7G8, effectively invade sialic acid-deficient erythrocytes. Type II parasites, represented by clones FCR-3/A2, Camp/A1, and Dd2, are dependent on erythrocyte sialic acid residues for invasion. These findings concur with the results of previous studies $(8-10,13)$.

In the course of the present investigations we have found that type II parasites are separable into two distinct subtypes (types II-A and II-B). Type II-A parasites are unable to survive when propagated in neuraminidase-treated erythrocytes. Type II-B parasites produce parasite lines that are able to invade and grow equally well in untreated and neuraminidase-treated erythrocytes. Clone Dd2, a type II-B parasite, produced such populations in repeated experiments of propagation in neuraminidase-treated erythrocytes. Further, four subclones of Dd2 likewise yielded sialic acid-independent lines. From these data we infer that each and every parasite of the Dd2 population carries the potential to produce non-sialic acid dependent lines under selection pressure. Unlike the type I parasites that 


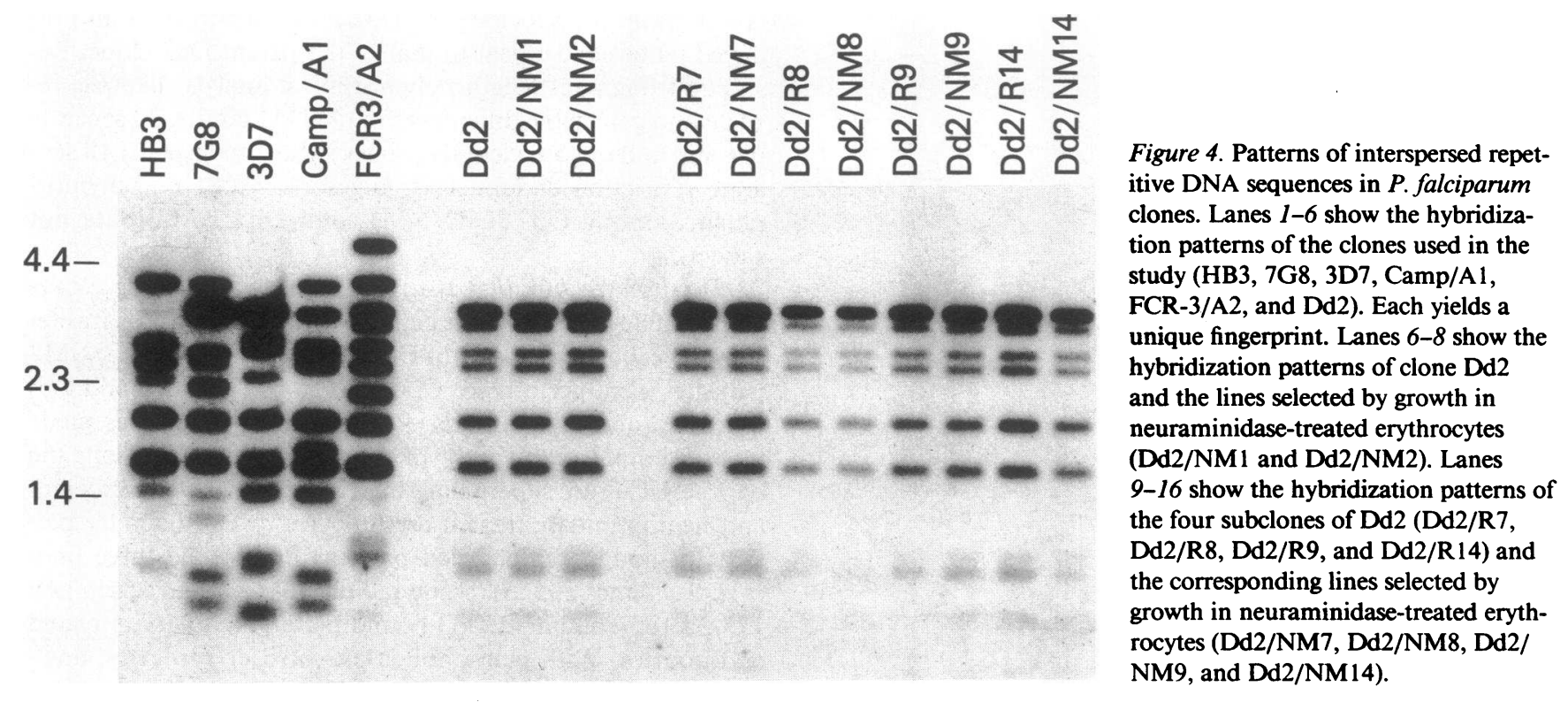

invade neuraminidase-treated erythrocytes at subnormal rates, non-sialic acid dependent lines derived from $\mathrm{Dd} 2$ invade both untreated and neuraminidase-treated erythrocytes equally well.

Experiments with an uncloned isolate, Thai-2, previously demonstrated that non-sialic acid dependent parasites could be selected from a parasite population (8). Propagation of the Thai-2 isolate for a period of time in Tn erythrocytes (deficient in the galactose and sialic acid components of the glycophorin oligosaccharide side chain [11]) yielded "Thai-Tn" parasites that were characterized by an enhanced ability to invade neuraminidase-treated erythrocytes. In the course of the present work, we found Thai- 2 by restriction fragment length polymorphism analysis to be polyclonal in character (data not shown). Hence, it is uncertain if type II-B parasites were present in Thai-2 or if propagation in neuraminidase-treated erythrocytes selected a subpopulation of type I parasites.

Erythrocyte binding assays were performed in an attempt to identify any differentially expressed proteins that might be associated with the ability to invade neuraminidase-treated erythrocytes. We were unable to demonstrate any differences among Dd2 and Dd2/NM1 or Dd2/NM2. Several possible explanations might account for this lack of discrimination, including inability to solubilize parasite components with our procedures, insufficient protein labeling, or involvement of nonprotein parasite components in the invasion process. A 175-kD binding protein described by Camus and Hadley (13) was able to bind untreated erythrocytes but not neuraminidase-treated erythrocytes. Mutational events involving this protein are not likely to explain the non-sialic acid dependent invasion of Dd2/NM.

One hypothesis that may account for the adaptation process is that the $\mathrm{Dd} 2$ population is continually switching between non-sialic acid dependent and sialic acid-dependent phenotypes. The ability of organisms to switch phenotypes is a well-described biologic phenomenon including antigenic variation in bacteria and protozoa, mating types in yeast, and immunoglobulin heavy chains in vertebrates (30-34). Genetic switch mechanisms expand the biologic repertoire of organ- isms and permit adaptive capacity in changing environments.

The suggestion that $\mathrm{Dd} / \mathrm{NM}$ might result from a genetic switch mechanism is based on two observations. First, the switched parasite acquires a positive attribute, the ability to invade an erythrocyte that the majority of the starting population is unable to invade. Second, all four subclones of Dd2 produced non-sialic acid dependent lines when propagated in neuraminidase-treated erythrocytes. These observations are reminiscent of the surface protein variations that have been described for Borrelia, Salmonella, and Trypanosome species (30-32). In these instances variation of surface proteins has been demonstrated to be the result of genetic switch mechanisms.

During cultivation in normal erythrocytes the $\mathrm{Dd} 2$ population may be switching at low frequency between the $\mathrm{Dd} 2$ and Dd2/NM phenotypes, but Dd2/NM phenotype would not develop because of the absence of selection pressure. The Dd2/ NM phenotype is seen when conditions, neuraminidasetreated erythrocytes, confer an advantage. Conversely, one might anticipate that there is switching between $\mathrm{Dd} 2 / \mathrm{NM}$ and $\mathrm{Dd} 2$ when $\mathrm{Dd} 2 / \mathrm{NM}$ is propagated in untreated erythrocytes. However, since Dd 2 and Dd2/NM grow at equivalent rates in untreated erythrocytes, there is no apparent advantage to Dd2. One could speculate that the removal of the non-sialic dependent pathway would result in the reappearance of the Dd2 phenotype. Bacterial and yeast plating techniques permit direct verification and quantitation of switching phenomenon. These techniques are not adaptable to malaria parasite cultures. Definitive proof of a switch mechanism must, therefore, await identification of the molecules involved in the non-sialic acid dependent pathway of invasion.

It will be of interest to examine freshly cloned clinical isolates for invasion pathway heterogeneity and the ability to switch phenotypes. To date investigations of $P$. falciparum invasion have used uncloned parasite populations or parasite clones that have been propagated for prolonged periods of time under in vitro culture conditions. Alterations in cytoadherence phenotype and the deletion of a parasite gene associated with cytoadherence in the human host have been asso- 

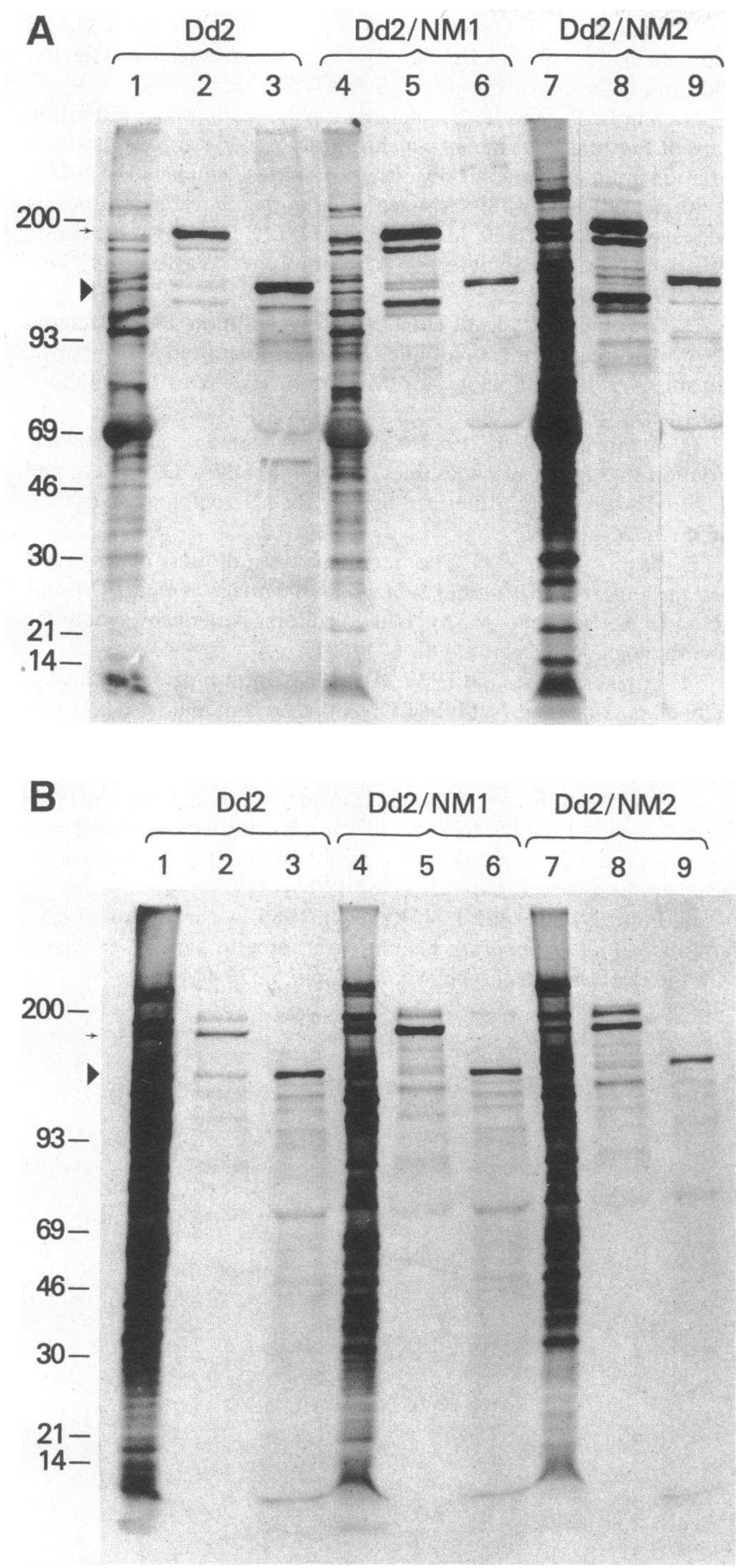

Figure 5. Erythrocyte binding assays of Dd2, Dd2/NM1, and Dd2/ NM2. $A$, Binding assays of soluble proteins from culture supernatants. Lanes 1,4 , and 7 show total radiolabeled proteins from culture supernatants of Dd2, Dd2/NM1, and Dd2/NM2, respectively. Lanes 2,5 , and 8 show radiolabeled protein bound to untreated erythrocytes. Lanes 3, 6, and 9 show radiolabeled protein bound to neuraminidase-treated erythrocytes. Arrow indicates $175 \mathrm{kD}$ band and arrowhead indicates $140-\mathrm{kD}$ band. $B$, Binding assays of proteins from corresponding parasite-infected cellular sonicates displayed as in $A$. Arrow indicates $165-\mathrm{kD}$ band and arrowhead indicates $140-\mathrm{kD}$ band.

ciated with in vitro propagation $(35,36)$. Similar phenomena may have occurred in the multi-step invasion process during in vitro propagation.
Dd2 and Dd2/NM parasites should be useful in delineating non-sialic acid dependent pathways of invasion. Identification of the molecules involved in this pathway may be possible through differential analysis, and would be predicted to contribute to our growing knowledge of the molecular events involving invasion.

\section{References}

1. Miller, L. H., J. D. Haynes, F. M. McCauliffe, T. Shiroshi, J. R. Durocher, and M. M. McGuinniss. 1977. Evidence for differences in erythrocyte receptors for the malarial parasite Plasmodium falciparum and Plasmodium knowlesi. J. Exp. Med. 146:277-281.

2. Perkins, M. 1981. Inhibitory effects of erythrocyte membrane proteins on the in vitro invasion of the human malaria parasite (Plasmodium falciparum) into its host cell. J. Cell Biol. 90:563-567.

3. Pasvol, G., M. Jungery, D. J. Weatherall, S. F. Parsons, D. J. Anstee, and M. J. Tanner. 1982. Glycophorin as a possible receptor for Plasmodium falciparum. Lancet. ii:947-950.

4. Cartron, J. P., O. Prou, M. Luilier, and J. P. Soulier. 1983. Susceptibility to invasion by Plasmodium falciparum of some human erythrocytes carrying rare blood group antigens. $\mathrm{Br}$. J. Haematol. 55:639-647.

5. Facer, C. A. 1983. Erythrocyte sialoglycoproteins and Plasmodium falciparum invasion. Trans. R. Soc. Trop. Med. Hyg. 77:524530 .

6. Breur, W. V., H. Ginsburg, and Z. I. Cabanchik. 1983. An assay of malaria parasite invasion into human erythrocytes: the effects of chemical and enzymatic modification of erythrocyte membrane components. Biochim. Biophys. Acta. 755:263-271.

7. Friedman, M. J., T. Blankenberg, G. Sensebaugh, and T. S. Tenforde. 1984. Recognition and invasion of human erythrocytes by malarial parasites: contribution of sialoglycoproteins to attachment and host specificity. J. Cell Biol. 98:1672-1677.

8. Mitchell, G. H., T. J. Hadley, M. H. McGuiniss, F. W. Klotz, and L. H. Miller. 1986. Invasion of erythrocytes by Plasmodium falciparum malaria parasites. Evidence for receptor heterogeneity and two receptors. Blood. 67:1519-1521.

9. Hadley, T. J., F. W. Klotz, G. Pasvol, J. D. Haynes, M. H. McGuiniss, Y. Okubo, and L. H. Miller. 1987. Falciparum malaria parasites invade erythrocytes that lack glycophorin A and B (MkMk). Strain differences indicate receptor heterogeneity and two pathways for invasion. J. Clin Invest. 80:1190-1193.

10. Perkins, M. E., and E. H. Holt. 1988. Erythrocyte receptor recognition varies in Plasmodium falciparum isolates. Mol. Biochem. Parasitol. 27:23-34.

11. Anstee, D. J. 1981. The blood group MNSs-active sialoglycoproteins. Semin. Hematol. 18:13-31.

12. Pasvol, G., J. S. Wainscoat, and D. J. Weatherall. 1982. Erythrocytes deficient in glycophorin resist invasion by the malaria parasite Plasmodium falciparum. Nature (Lond.). 297:64-66.

13. Camus, D., and T. J. Hadley. 1985. A Plasmodium falciparum antigen that binds to host erythrocytes and merozoites. Science (Wash. DC). 230:553-556.

14. Perkins, M. E., and L. J. Rocco. 1988. Sialic acid-dependent binding of Plasmodium falciparum merozoite surface antigen, Pf200, to human erythrocytes. J. Immunol. 141:3190-3196.

15. Wellems, T. E., A. M. J. Oduola, B. Fenton, R. Desjardins, L. J. Panton, and V. E. do Rosario. 1988. Chromosome size variation occurs in cloned Plasmodium falciparum on in vitro cultivation. Rev. Bras. Genet. 11:813-825.

16. Bhasin, V. K., and W. Trager. 1984. Gametocyte-forming and non-gametocyte-forming clones of Plasmodium falciparum. Am. J. Trop. Med. Hyg. 33:534-537.

17. Walliker, D., I. A. Quakyi, T. E. Wellems, T. F. McCutchan, A. Szarfman, W. T. London, L. M. Corcoran, T. R. Burkot, and R. Carter. 1987. Genetic analysis of the human malaria parasite Plasmodium falciparum. Science (Wash. DC). 236:1661-1666. 
18. Burkot, T. R., J. L. Williams, and I. Schneider. 1984. Infectivity to mosquitoes of Plasmodium falciparum clones grown in vitro from the same isolate. Trans. R. Soc. Trop. Med. Hyg. 78:339-341.

19. Trager, W., M. Tershakovec, L. Lyandvert, H. Stanley, N. Lanners, and E. Gubert. 1981. Clones of the malaria parasite Plasmodium falciparum obtained by microscopic selection: their characterization with regard to knobs, chloroquine sensitivity and formation of gametocytes. Proc. Natl. Acad. Sci. USA. 78:6527-6530.

20. Trager, W., and J. B. Jensen. 1976. Human malaria parasites in continuous culture. Science 193:673-675.

21. do Rosario, V. E. 1981. Cloning of naturally occurring mixed infections of malaria parasites. Science (Wash. DC). 212:1037-1038.

22. Lambros, C., and J. P. Vanderberg. 1979. Synchronization of Plasmodium falciparum erythrocytic stages in culture. J. Parasitol. 65:418-420.

23. Dluzewski, A. R., I. T. King, K. Rangachari, P. A. Bates, and R. J. Wilson. 1984. A simple method for isolating viable mature parasites of Plasmodium falciparum from cultures. Trans. $R$. Soc. Trop. Med. Hyg. 78:622-624.

24. Aley, S. B., J. A. Sherwood, and R. J. Howard. 1984. Knob-positive and knob-negative Plasmodium falciparum differ in expression of a strain-specific malarial antigen on the surface of infected erythrocytes. J. Exp. Med. 160:1585-1590.

25. Haynes, J. D., J. P. Dalton, F. W. Klotz, M. H. McGuiniss, T. J. Hadley, D. E. Hudson, and L. H. Miller. 1988. Receptor-like specificity of a Plasmodium knowlesi malarial protein that binds to Duffy antigen ligands on erythrocytes. J. Exp. Med. 167:1873-1881.

26. Siddiqui, W. A., S. C. Kan, K. Kramer, and S. M. RichmondCrum. 1979. In vitro production and partial purification of Plasmodium falciparum antigen. Bull. WHO. 57:75-82.

27. Maniatis, T., E. F. Fritsch, and J. Sambrook. 1982. Molecular Cloning: A Laboratory Manual. Cold Spring Harbor Laboratory, Cold Spring Harbor, NY. 545 pp.
28. Rigaud, G., T. Grange, and R. Pictet. 1987. The use of $\mathrm{NaOH}$ as transfer solution of DNA onto nylon membrane decreases the hybridization efficiency. Nucleic Acids Res. 15:857.

29. Sinnis, P., and T. E. Wellems. 1988. Long range restriction maps of Plasmodium falciparum chromosomes: crossingover and size variation among geographically distant isolates. Genomics. 3:287-295.

30. Barbour, A. 1989. Antigenic variation in relapsing Borrelia species: genetic aspects. In Mobile DNA. D. E. Berg and M. M. Howe, editors. American Society for Microbiology, Washington, DC. 783-789.

31. Glasgow, A. C., K. C. Hughes, and M. I. Simon. 1989. Bacterial DNA inversion systems. In Mobile DNA. D. E. Berg and M. M. Howe, editors. American Society for Microbiology, Washington, DC. 637-660.

32. Donnelson, J. E. 1989. DNA rearrangements and antigenic variation in African trypanasomes. In Mobile DNA. D. E. Berg and M. M. Howe, editors. American Society for Microbiology, Washington, DC. 763-781.

33. Klar, A. J. S. 1989. The interconversion of yeast mating type: Saccharomyces cerevisiae and Schizosaccharomyces pombe. In Mobile DNA. D. E. Berg and M. M. Howe, editors. American Society for Microbiology, Washington, DC. 671-691.

34. Lutzker, S. G., and F. W. Alt. 1989. Immunoglobulin heavychain class switching. In Mobile DNA. D. E. Berg and M. M. Howe, editors. American Society for Microbiology, Washington, DC. 693-714.

35. Magowan, C., W. Wollish, L. Anderson, and J. Leech. 1988. Cytoadherence by Plasmodium falciparum-infected erythrocytes is correlated with the expression of a family of variable proteins on infected erythrocytes. J. Exp. Med. 168:1307-1319.

36. Pologe, L. G., and J. V. Ravetch. 1986. A chromosomal rearrangement in $P$. falciparum histidine-rich protein gene is associated with the knobless phenotype. Nature (Lond.). 322:474-477. 